\title{
Der Skutari-See: einzigartiges Naturjuwel in Montenegro
}

\author{
Hilke Steinecke \& Peter Schubert
}

\begin{abstract}
Lake Skadar is located in the southeast of Montenegro. It is the largest lake in the Balkans. Two thirds of the area of the lake are located in Montenegro. This part has been a national parc since 1983. It is an unique inland water ecosystem. The northern extensive floating leaf zones with white water lily and water caltrop and the adjacent marshland part comprises flooded in spring. The lake offers habitat for many water birds including the rare dalmatian pelican. Different habitats at the lake with their flora and fauna are presented.
\end{abstract}

\section{Zusammenfassung}

Der Skutari-See liegt im Südosten von Montenegro und ist der größte See des Balkans. Zwei Drittel der Fläche des Sees liegen in Montenegro. Dieser Teil ist seit 1983 als Nationalpark ausgewiesen. Es handelt sich um ein einzigartiges Binnengewässer-Ökosystem. Charakteristisch sind im Norden des Sees die ausgedehnten Schwimmblattzonen mit Weißer Seerose und Wassernuss sowie das angrenzende Marschland. Im Frühling ist es überschwemmt. Der See bietet vielen Wasservögeln Lebensraum, so auch dem seltenen Krauskopf-Pelikan. Verschiedene Lebensräume am See mit ihrer Pflanzen- und Tierwelt werden vorgestellt.

\section{Einleitung}

Montenegro ist ein kleiner Balkanstaat an der Adria, der von Kroatien, Bosnien-Herzegowina, Serbien, dem Kosovo und Albanien umgeben wird. Nach dem Zerfall Jugoslawiens erhielt Monte-

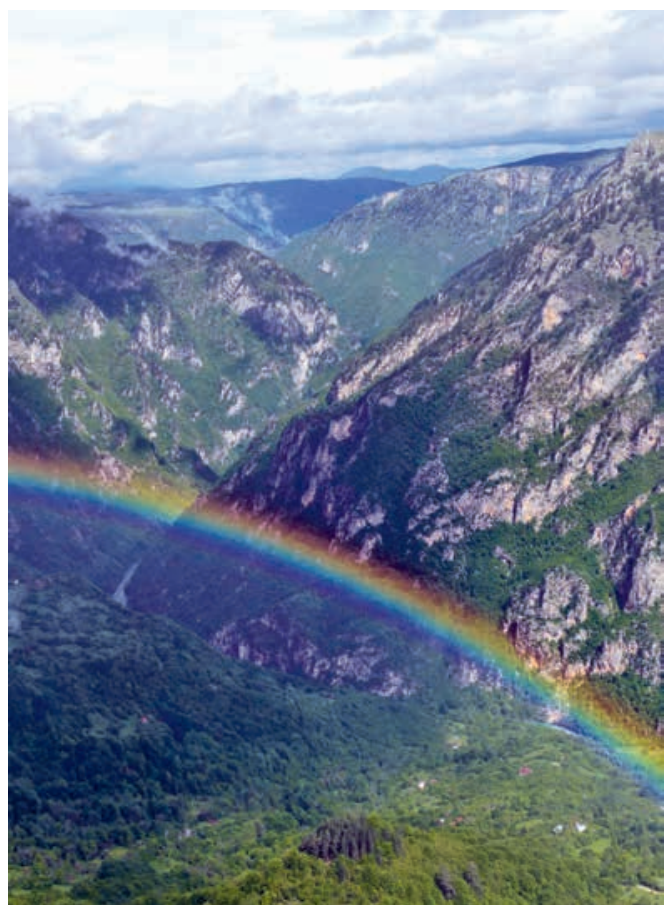

Abb. 1: Montenegro ist ein sehr gebirgiges Land mit vielen Flüssen und Seen. Hier ein Blick auf die Tara-Schlucht im Nationalpark Durmitor. (Foto: H. STEINECKE) negro im Jahre 2006 seine vollständige Unabhängigkeit. Mit einer Fläche von rund $14000 \mathrm{~km}^{2}$ ist Montenegro etwas kleiner als Schleswig-Holstein. Die Einwohnerzahl liegt bei rund 640000 Personen, das sind weniger Einwohner als in Frankfurt, dementsprechend dünn besiedelt ist das Land und gibt es noch reichlich Natur zu entdecken. Die meisten Menschen leben in der Hauptstadt Podgorica. Vor dem Balkankrieg war Montenegro als Teil Jugoslawiens ein beliebtes touristisches Ziel, in Folge des Krieges sanken naturgemäß die Touristenzahlen deutlich. Nun wird Montenegro als Urlaubsland wieder zunehmend beliebter, der Staat fördert den Ausbau touristischer Infrastruktur, vor allem auch in den Wintersportgebieten in den Bergen im Norden. Während an der Adriaküste die Strände durch den Bau von touristischen Massenunterkünften abschnittsweise verschandelt wurden, gibt es im Inland noch immer eine großartige, sehr ursprüngliche Natur zu erkunden.

Der größte Teil Montenegros ist Gebirgsland, es handelt sich um den südöstlichen Teil des Dinarischen Gebirges. Die Hochgebirgslandschaft ist schroff und von tiefen Schluchten durchzogen, die imposante Tara-Schlucht gilt als die tiefste Schlucht Europas. Nur im Südosten Montenegros befinden sich Niederungen, in denen die Hauptstadt Podgorica sowie der Skutari-See liegen. 


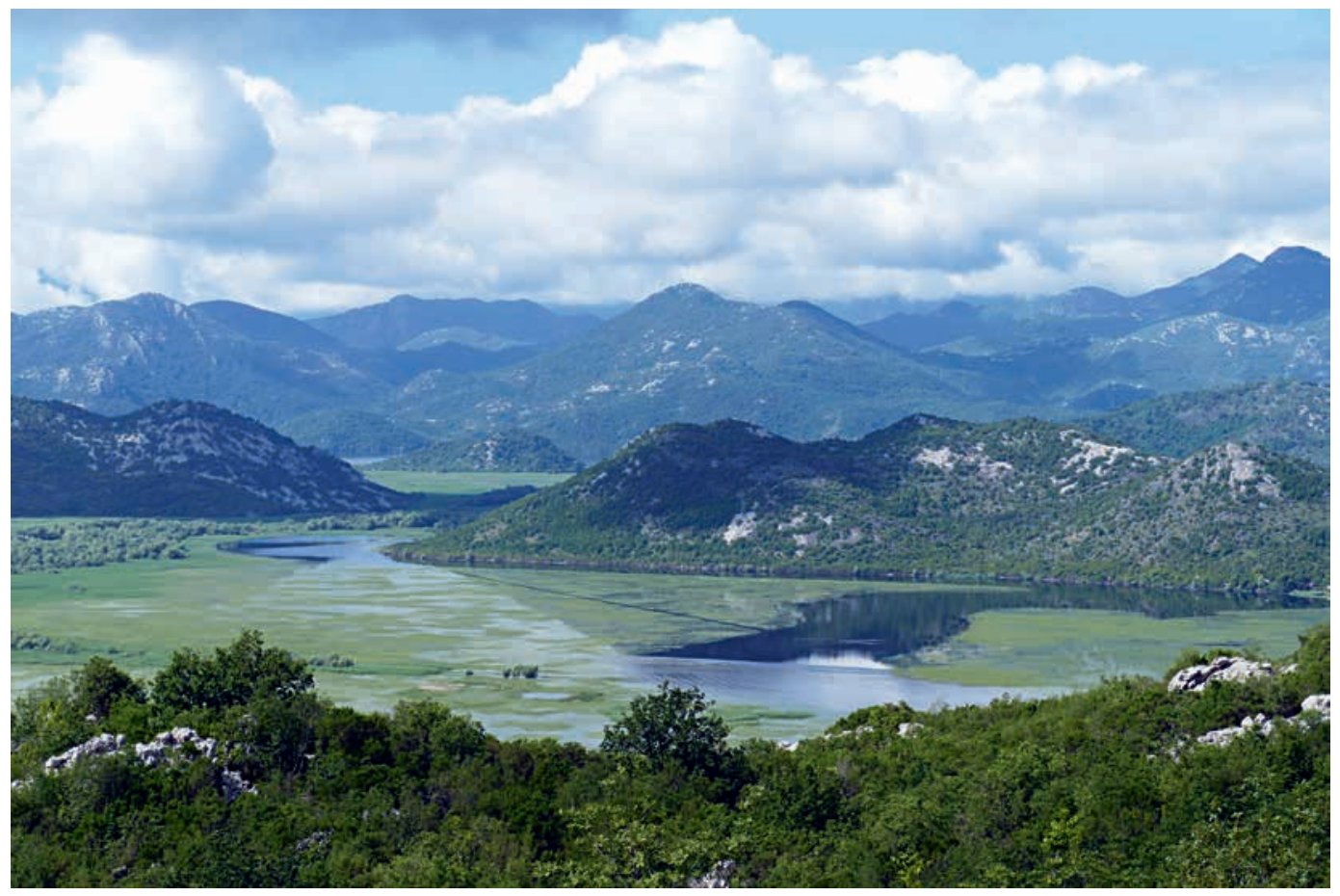

Abb. 2: Blick über das Nordende des Skutari-Sees auf das Dorf Dodoši mit seinen ausgedehnten Schwimmblattzonen (hellgrün) und Beständen der Silber-Weide (dunkelgrün). (Foto: H. Steinecke)

Montenegro ist sich seiner großartigen Natur bewusst: das kleine Land beherbergt immerhin fünf Nationalparks: neben Biogradska Gora, Durmitor und Lovćen (alle drei gegründet 1952) sowie Prokletije (gegründet 2009) auch den Nationalpark Skutari-See. Letzterer ist mit einer Fläche von 40000 ha der größte Nationalpark des Landes. Insgesamt ist der See $48 \mathrm{~km}$ lang und $14 \mathrm{~km}$ breit und wird vom bis $1600 \mathrm{~m}$ hohen Rumija-Gebirge umgeben, was landschaftlich äußerst reizvoll ist. Zwei Drittel seiner Fläche liegen in Montenegro, der Rest gehört zu Albanien. Die Küstenlinie in Montenegro beträgt je nach Wasserstand 110-168 km, in Albanien sind es etwa $57 \mathrm{~km}$. Zuerst (1983) wurde der See in Montenegro unter Schutz gestellt und als Nationalpark ausgewiesen, 1996 wurde er zusätzlich in die internationale Ramsar-Liste schützenswerter Feuchtgebiete aufgenommen. Der albanische Teil des Sees wurde erst 2005 als Naturreservat ausgewiesen.

\section{Der Skutari-See}

Der See wurde nach der in Albanien am Seeufer gelegenen Stadt Shkodra (auch Shkodë) Liqen i Shkodrës benannt, in Montenegro heißt er Skadarsko jezero und auf englisch Skadar lake. Der italienische, auch im Deutschen verwendete Name Skutari-See (oder auch Scutari-See) nimmt Bezug auf die italienische Bezeichnung der Stadt Shkodra. Erdgeschichtlich ist der See noch recht jung.

Während der letzten Eiszeit waren die Berge im Bereich des heutigen Montenegros besonders stark vergletschert. Schmelzwasser und Gletscherflüsse prägten die Ebene, bevor sich dort vor etwa 18000 Jahren der Skutari-See bildete. Er liegt in einem sogenannten Polje, das ist eine ausgedehnte, allseits geschlossene Hohlform im Karst mit zumeist ebenem Boden und stellenweise steiler Umrahmung durch Berge. Gespeist wird der Skutari-See von zahlreichen, bis $60 \mathrm{~m}$ tief reichenden Karstquellen am Grunde des Sees. Diese sogenannten Augen oder Okas sind für die besondere Artenvielfalt 


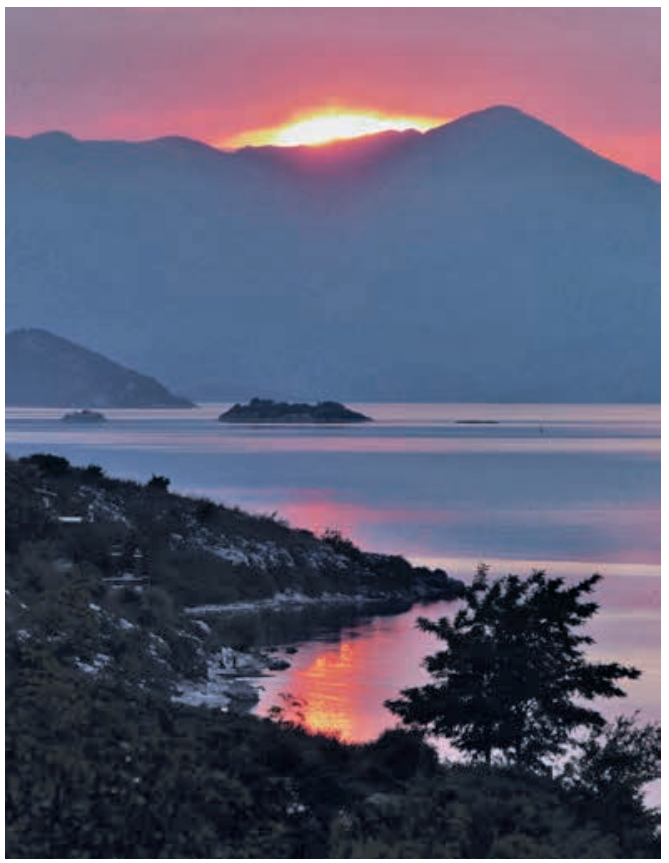

Abb. 3: Blick über den Skutari-See von Albanien (Nähe Shkodra) in Richtung Montenegro. Hier fehlen die ausgedehnten Seerosen-Teppiche (Foto: H. STeinecke)

im See verantwortlich, da über sie ständig frisches, sehr sauberes Wasser in den See strömt. Außerdem wird der See von Flüssen aus den umliegenden Bergen gespeist. Wichtigster Zufluss



Abb. 4: Ende Mai waten die Rinder im Schwemmland mitunter noch knietief durch das Wasser, die Wege sind unpassierbar. (Foto: H. Steinecke) ist die Morača, die Entwässerung des Sees erfolgt über den kleinen Fluss Buna in die nur $20 \mathrm{~km}$ entfernte Adria. Wenn der See im Frühjahr nach der Schneeschmelze seinen höchsten Wasserstand hat, umfasst er eine Fläche von rund $600 \mathrm{~km}^{2}$ und ist somit vergleichbar mit dem Bodensee und der größte See des Balkans. Allerdings schwankt der Wasserstand stark. Denn das Klima ist submediterran mit milden, regenreichen Wintern und heißen trockenen Sommern, in denen durchaus $40{ }^{\circ} \mathrm{C}$ erreicht werden. Nach dem Sommer ist so viel Wasser verdunstet, dass die Fläche des Sees im Herbst auf rund $400 \mathrm{~km}^{2}$ schrumpft.

Im Gegensatz zum Bodensee ist der Skutari-See aber nur wenige Meter tief. Dementsprechend erwärmt sich das Wasser im Sommer auf etwa $30^{\circ} \mathrm{C}$. Im und am warmen Wasser haben sich zahlreiche Pflanzen und Tiere angesiedelt, darunter etwa 280 Vogelarten, die hier im Winter als Rast- oder im Sommer als Brutvögel vorkommen. Im Bereich des Sees gibt es etwa 20 endemische Tier- und Pflanzenarten. Wichtige Nahrungsgrundlage für die reiche Vogelwelt sowie die vielen Fische im See sind die zahlreichen Amphibien und die vielen Insekten. Während bei uns das Insektensterben dramatische Ausmaße annimmt, sind hier Insekten bezüglich ihrer Arten- und Individuenzahl üppig vorhanden. Faszinierend ist es zu beobachten, wel-

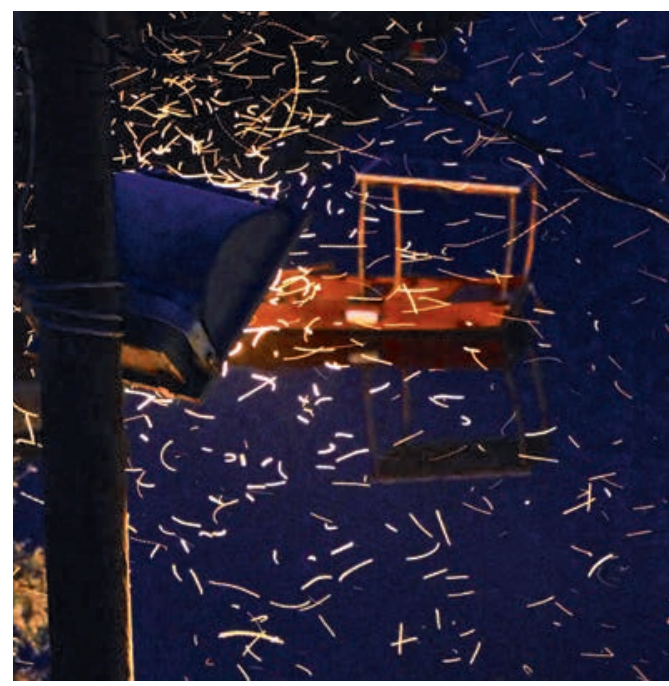

Abb. 5: Wie ein kleines Feurwerk schwirren die vielen Insekten im Licht einer Straßenlaterne. (Foto: H. Steinecke) 


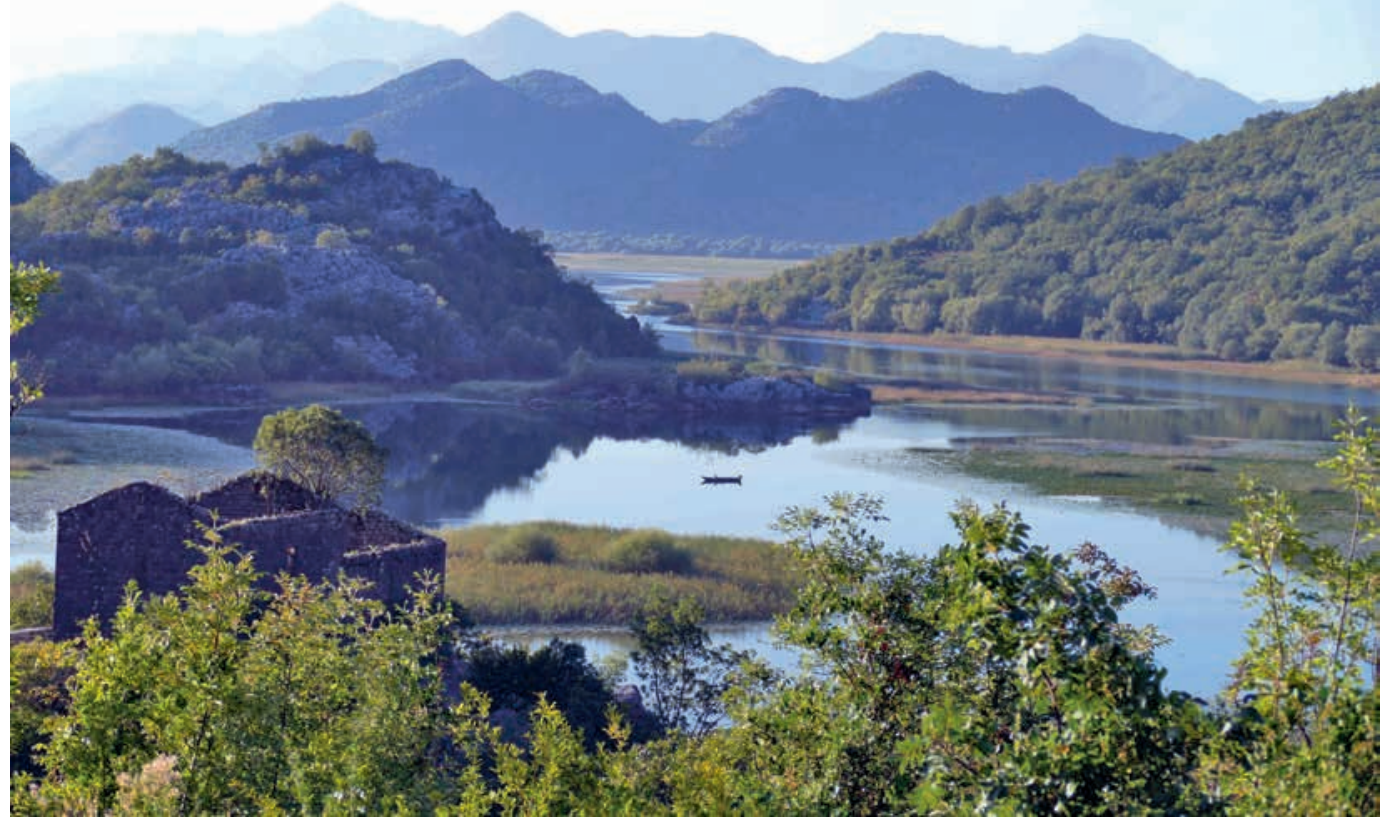

Abb. 6: Blick von Karuç aus über den Skutari-See, im Vordergrund die Ruine von St. Petar. (Foto: H. STEInEcke)

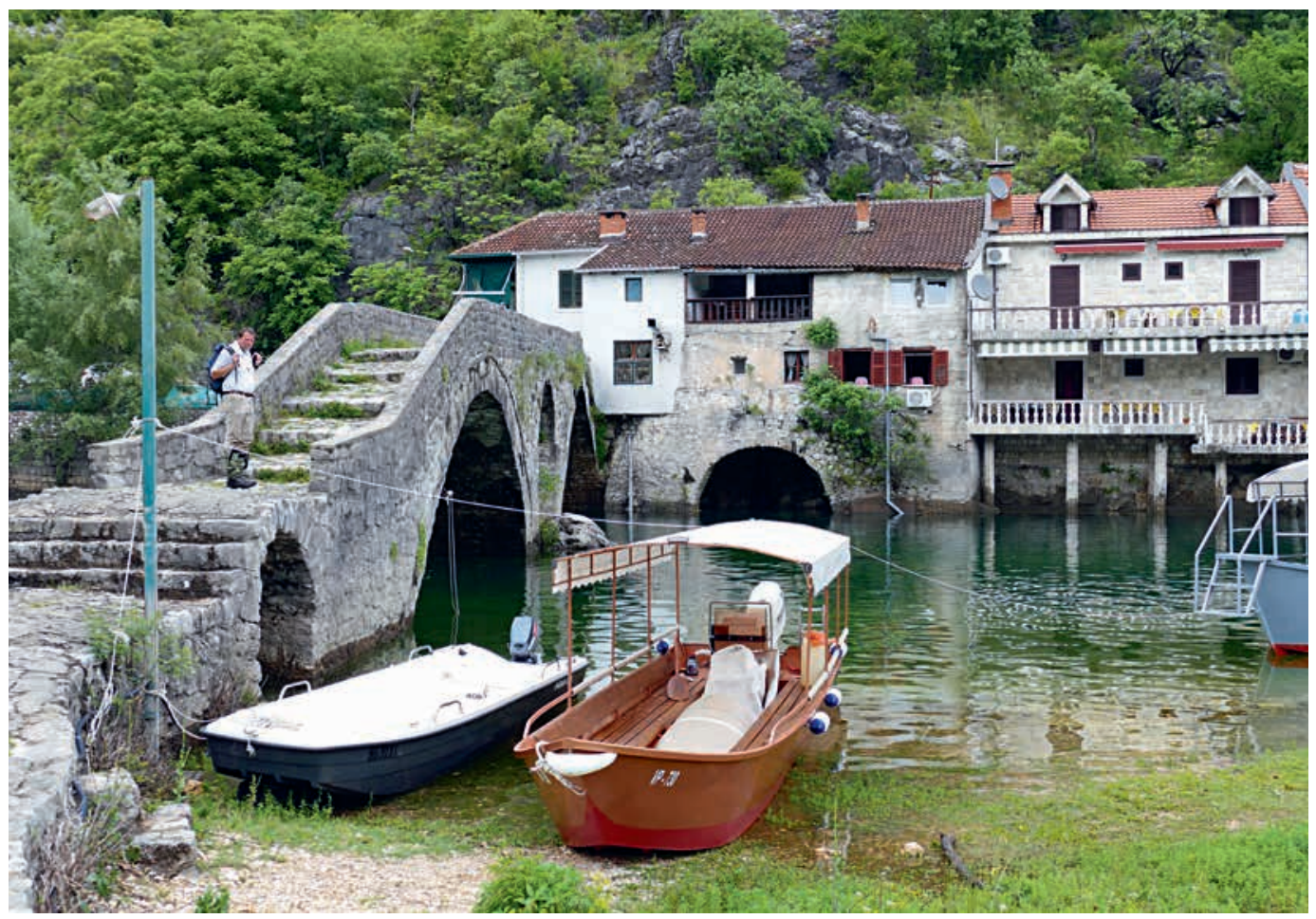

Abb. 7: Rijeka Crnojevića. (Foto: H. Steinecke) 


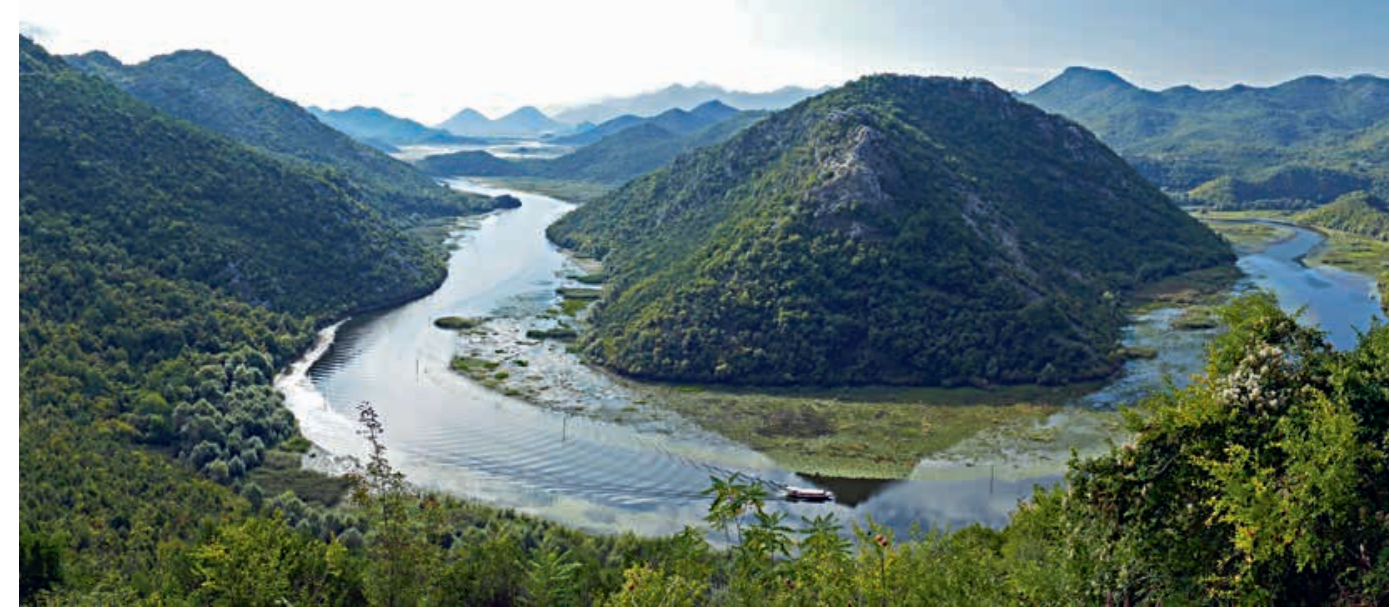

Abb. 8: Umlaufschleife des Zuflusses Crnojevića. (Foto: H. Steinecke)

che Insektenmassen sich abends im Lichtschein von Laternen oder Lampen an Häusern in der Nähe des Seeufers tummeln.

Im Bereich des Sees gibt es verschiedene Lebensräume mit ihren speziellen Tier- und Pflanzenarten. Mit seinen ausgedehnten Sumpfgebieten und Schwimmblattzonen ist der Skutari-See ein äußerst faszinierendes Ökosystem. Nachfolgend werden unterschiedliche Lebensräume vorgestellt. Die verschiedenen Jahreszeiten haben ihre ganz unterschiedlichen Aspekte, was auch an der unterschiedlichen Höhe des Wasserstandes liegt.

\section{Schwimmblattzone}

Im Norden ist der See durch viele Buchten und Quellen geprägt. Es gibt zwar eine Uferstraße entlang des Sees, nicht immer ist es aber möglich, direkt bis an das Ufer vorzudringen. Auch sind auf Wanderkarten noch Fußwege verzeichnet, die mitunter mittlerwiele aber vernachlässigt wurden und zugewachsen sind, so dass sie nicht mehr nutzbar sind. Besonders schwierig ist das Erreichen des Seeufers im Frühling bei hohem Wasserstand. Einen guten Überblick über den See und die ausgedehnten Schwimmblattzonen kann man sich am besten verschaffen, wenn man die kleinen alten, direkt am See gelegenen Orte aufsucht. Ein besonders pittoresker, bereits im 15. Jahrhundert erwähnter Fischerort ist Karuç mit seinen alten, z. T. verfallenen Steinhäusern. Über dem Ort thront auf einem kleinen Felsen die Ruine der kleinen Kirche St. Petar. Von oberhalb des Ortes ergeben sich schöne Blicke über den Norden des See bis auf die hohen Berge in Albanien.

Ein sehr geschichtsträchtiger Ort ist auch das um 1481 gegründetet Städtchen Rijeka Crnojevića mit seiner alten Steinbrücke. Rijeka bedeutet Fluss, der Ort liegt ja auch am gleichnamigen Fluss Crnojevića. Hier, wo er den See speist, zeigt er eine beeindruckende Schleife, deren Abbild als Symbol für die Schönheit von Montenegros Natur Reiseführer und Landkarten schmückt.

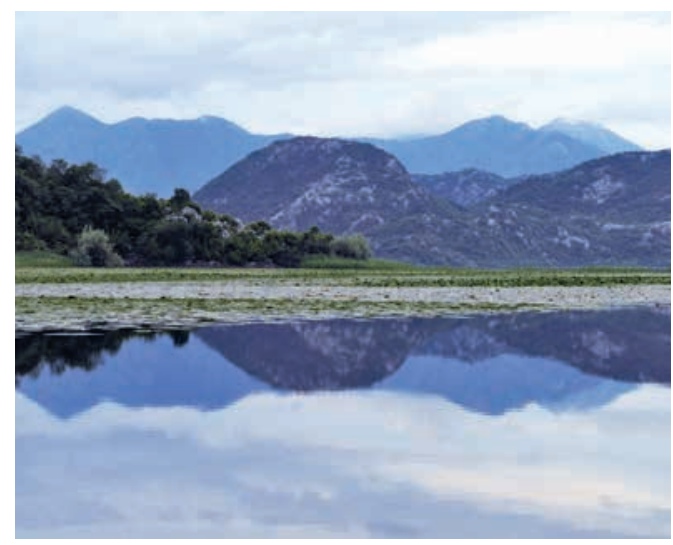

Abb. 9: Die umliegenden Berge spiegeln sich auf dem See. (Foto: H. STEINecke) 


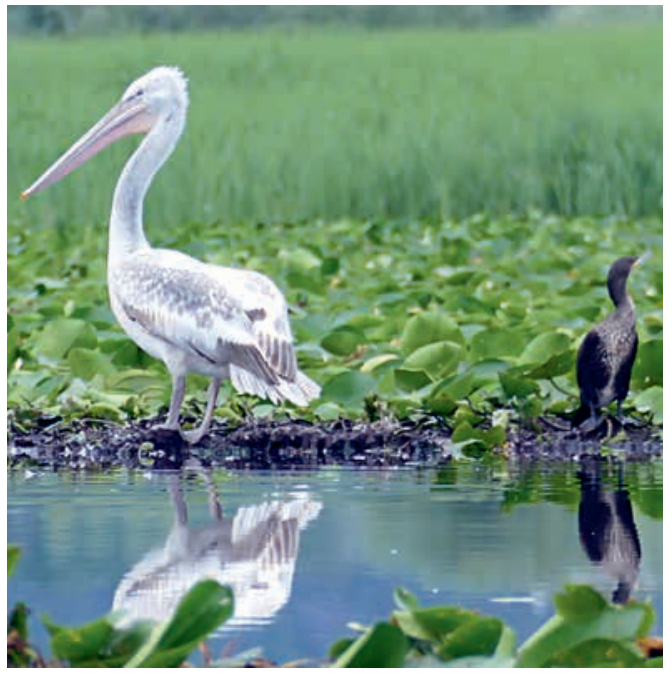

Abb. 10: Krauskopf-Pelikan und Kormoran in der Schwimmblattzone. (Foto: H. Steinecke)

Von beiden Orten kann man die ausgedehnten Schwimmblattzonen auf dem Wasser erkunden. Sie bedecken über $30 \mathrm{~km}^{2}$ der Seeoberfläche, was für Europa einmalig ist. In Massen tritt hier die echte Weiße Seerose (Nymphaea alba) auf, die in Deutschland mittlerweile sehr selten geworden ist. Dazwischen wächst die Gelbe Teichrose (Nuphar lutea). Faszinierend sind auch die Unmengen der Wassernuss (Trapa natans), die in Deutschland vom Aussterben bedroht ist. Charakteristisch für die Schwimmblattpflanze mit ihren rhombischen Blättern sind ihre harten Nussfrüchte, die mit zwei bis vier Dornen, die aus den Kelchblättern hervorgehen, ausgestattet sind. Wenn die reifen und von der Mutterpflanze losgelösten Früchte auf den Seeboden sinken, dienen diese Dornen der Verankerung. Die stärkehaltigen Früchte wurden früher gegessen. Sie müssen allerdings vor dem Verzehr gekocht werden, da sie roh giftig sind. Im Herbst kann man taube Nüsse im Uferbereich des Sees in großen Massen angeschwemmt finden.

Bei ruhigem Wetter entsteht vom Boot auf dem See aus betrachtet der Eindruck, sich auf einem Spiegel zu befinden, wenn sich auf der Wasseroberfläche das Spiegelbild der umliegenden Berge abbildet. Auf dem See leben viele Wasservögel wie beispielsweise Kormorane oder Seeschwalben. Das Juwel unter den Vögeln ist der Krauskopf-Pelikan

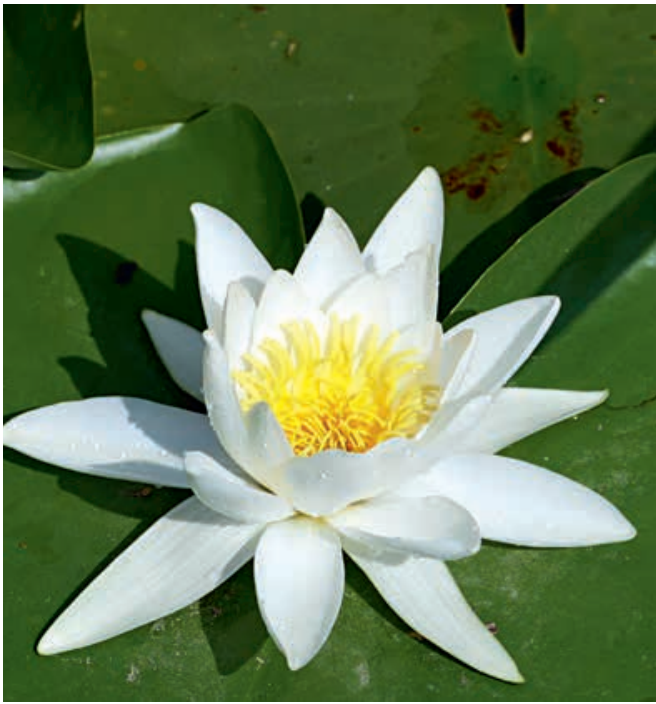

Abb. 11: Weiße Seerose (Nymphaea alba). (Foto: H. Steinecke)

(Pelicanus crispus), der von Südost-Europa bis zur Mongolei heimisch ist. Hier befindet sich noch eine der wenigen Brutkolonien in Europa. Aufgrund der Zerstörung seines Lebensraumes durch Trockenlegen von Feuchtgebieten ist dieser Pelikan, der größer als ein Schwan ist, bedroht und streng geschützt. Am Skutari-See konnte sich die Population etwas erholen, hier gibt es nun wieder etwa 100 Tiere. Krauskopf-Pelikane brüten am Skutari-See wieder seit 2014

Das Leben am See ist noch ziemlich ursprünglich, Fischer gehen im Idealfall mit einfachen Reusen in kleinen Booten auf den Fischfang. Wichtigste Speisefische sind hier Karpfen, Aale, Lachsforellen und Hechte.

\section{Sümpfe, Marschland und Auwälder}

Die ausgedehnte Sumpf- und Auenlandschaft lässt sich erst bei niedrigem Wasserstand am Ende des Sommers erkunden. Eine Besonderheit der Verlandungszone besteht darin, dass sich unter dem Schilf Torfinseln befinden. Auf diesen wachsen Silber-Weiden (Populus alba) einzeln oder in Gruppen, so dass die Landschaft wie eine weitläufige Parklandschaft wirkt. Das trockengefallene Schwemmland wird in der zweiten Jahreshälfte als extensives Weideland für Rinder, Pferde, Ziegen oder Schafe genutzt. 


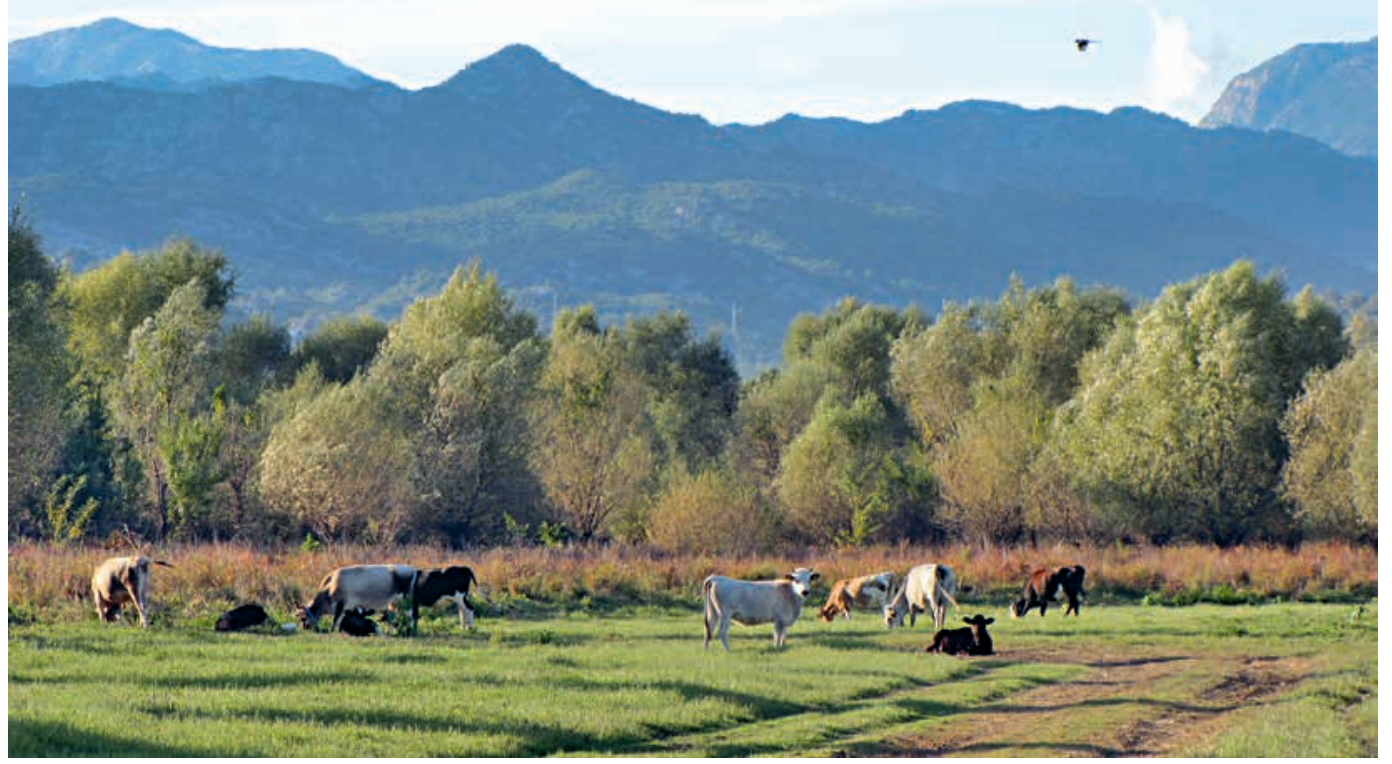

Abb. 12: Als Weideland genutztes Schwemmland mit Silber-Weiden. (Foto: H. Steinecke)

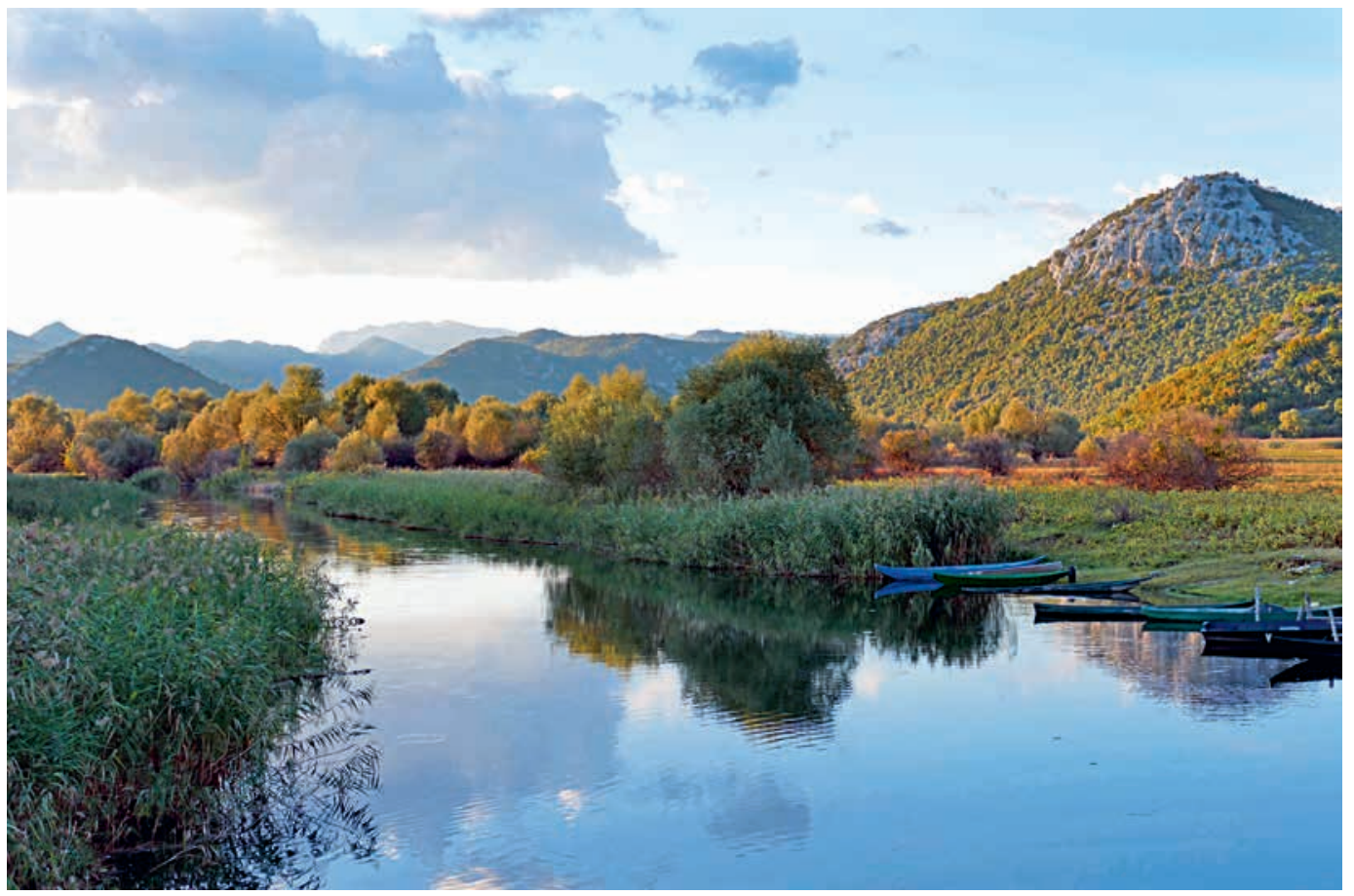

Abb. 13: Die Ufer der Zuflüsse sind von Schilfrohr gesäumt. (Foto: H. Steinecke) 


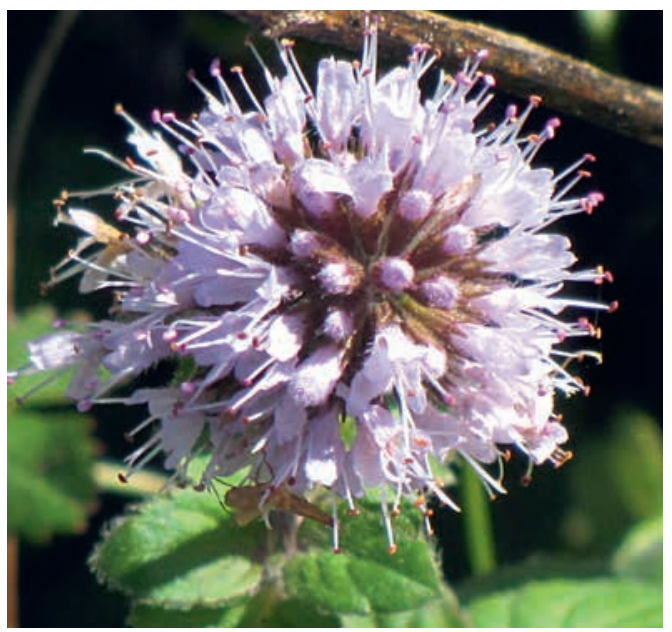

Abb. 14: Wasser-Minze (Mentha aquatica).

(Foto: H. STEINeCKe)

Auf verschiedenen landwirtschaftlich genutzten Pfaden kann man das Gelände durchstreifen. Im Herbst blühen hier Wasserminze (Mentha aquatica) und Flohkraut (Pulicaria dysenterica). Flächendeckend hat sich hier die Spitzklette (Xanthium strumarium), ein Neophyt aus Nordamerika, breit gemacht.Es gibt hier aber auch ein paar Spezialitäten wie das Lockerblütige Knabenkraut (Orchis laxiflora).

In den austrocknenden Pfützen im Schlamm findet man unzählige Exemplare des SkutariWasserfrosches (Pelophylax shqiperica). Oft sind die Tiere durch einen hellen Rückenstreifen gekennzeichnet. Ihren Namen erhielt die Art von ihrem wichtigen Verbreitungsgebiet am SkutariSee. Der Frosch kommt nur in Montenegro und Albanien vor. Er ist ein wichtiges Glied in der Nahrungskette. Noch scheint es viele Exemplare zu geben, doch sind die Bestände rückläufig durch Verschmutzung seiner Lebensräume oder durch Verdrängung durch eingeführte andere, konkurrenzstärkere Wasserfrösche. Im See gibt es zudem eine große Population der Würfelnatter (Natrix tesselata), die im Uferbereich auf Fischfang geht.

Der Norden des Sees ist nicht nur floristisch und faunistisch sehr interessant, er hat für die Region auch eine große kulturgeschichtliche Be-

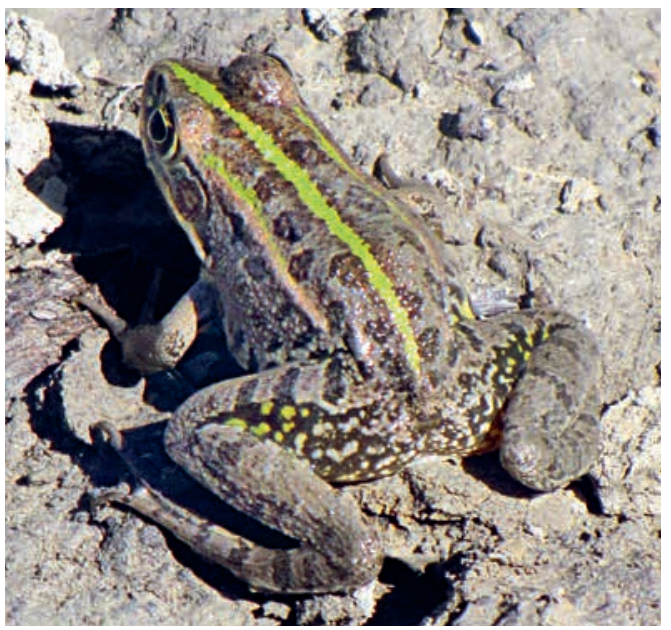

Abb. 15: Skutari-Wasserfrosch (Pelophylax shqiperica).

(Foto: H. Steinecke)

deutung. Gut geschützt durch den See wurden am Ufer oder auf Inseln einige Klöster gebaut. Malerisch erhebt sich aus dem Sumpf in der Nähe des Dorfes Dodoši die Ruine der mittelalterlichen Festung Žabljak Crnojevića. Hier residierte die Herrscherdynastie Crnojević, bis sie im 15. Jh. durch die Osmanen vertrieben wurde und die Hauptstadt Cetinje gründete.

\section{Macchie, Felsheiden und Mauern}

Im Gebiet rund um den Skutari-See gab es früher ausgedehnte Wälder, vor allem aus wärme- und

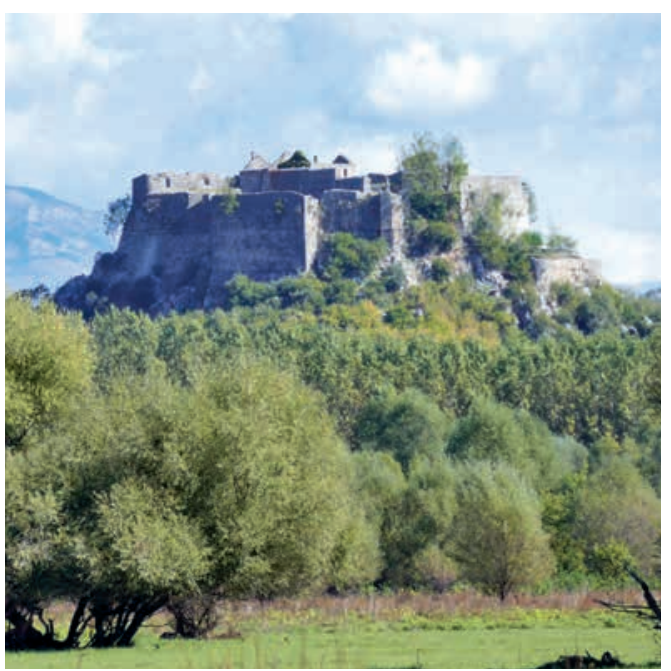

Abb. 16: Festung Žabljak Crnojevića. (Foto: H. Steinecke) 


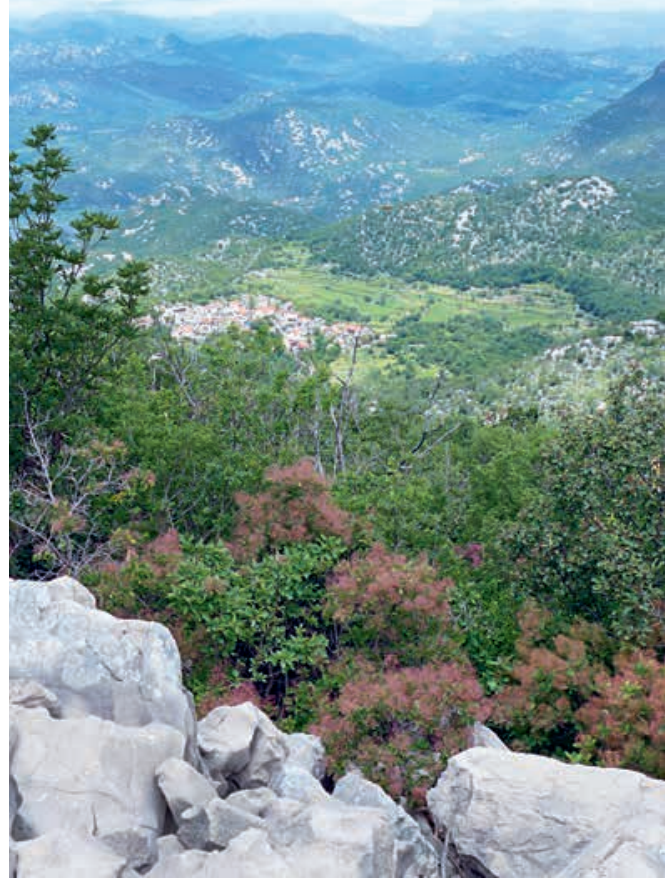

Abb. 17: Auf felsigen, trockenen Standorten gedeiht der Perückenstrauch (Cotinus coggygria). (Foto: H. STEINecke)

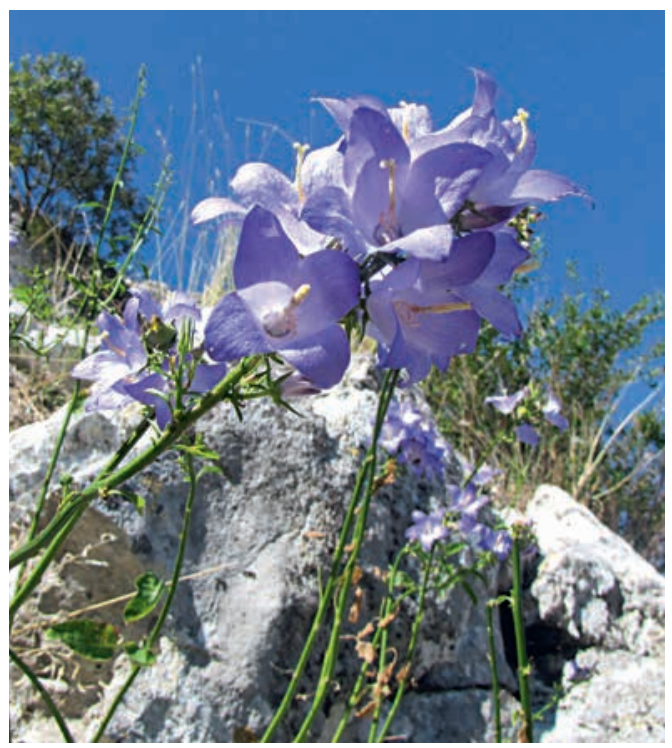

Abb. 18: Die Pyramiden-Glockenblume (Campanula pyramidalis) findet sich häufig an Mauern und Felsen.

(Foto: H. STeinecke) trockenheitsliebenden Eichen. Von den großen Beständen der endemischen Skutari-Eiche (Quercus robur subsp. scutariensis), einer Unterart unserer Stiel-Eiche, sind nur noch Reste erhalten. Die Wälder wurden übernutzt und zur Holzgewinnung abgeholzt. Als Ersatzgesellschaft traten Strauchformationen (Macchie) auf. Hier bilden zahlreiche Sträucher oder kleine Bäume oft undurchdringliche Bestände, darunter Stein-Eiche (Quercus ilex), Zistrosen (Cistus spp.) und Granatapfelbäume (Punica granatum). Die wilden Granatapfelbäume haben nur relativ kleine Früchte, die höchstens zur Saftgewinnung genutzt werden. In den Gärten werden meist die großfrüchtigen Kultursorten angebaut. Sehr kratzig ist die Schlingpflanze Tamus communis. An den steileren Hängen des Ufers gedeihen auch Christusdorn (Paliurus spina-christi), Perückenstrauch (Cotinus coggygria), Blasenstrauch (Colutea arborescens), Orientalische Hainbuche (Carpinus orientalis) und Hopfenbuche (Ostrya carpinifolia). All diese genannten Gehölze werden bei uns als Zierpflanzen kultiviert.

Dazwischen wächst manchmal die Italienische Waldrebe (Clematis viticella), deren violette Blüten noch im Herbst zu finden sind. Ähnliches Arteninventar zeigen auch die Felsheiden, die sich auf

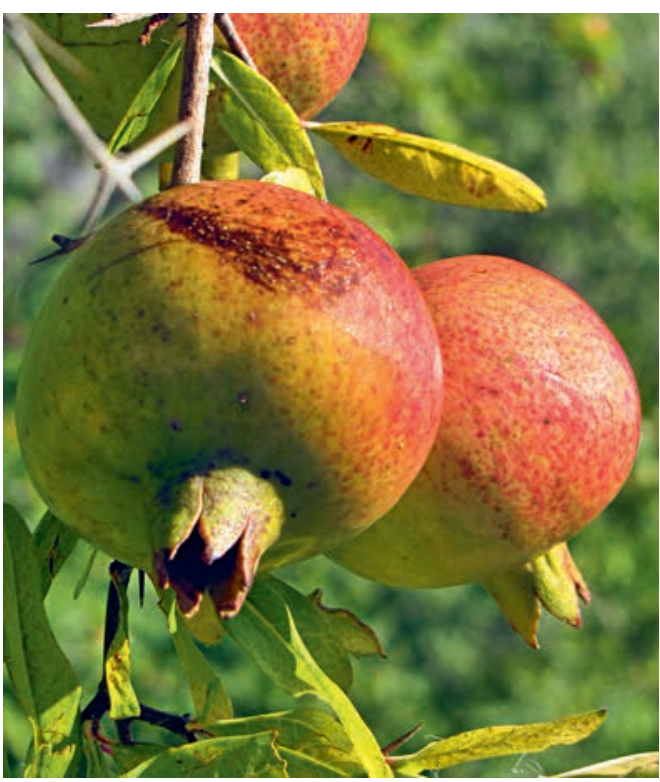

Abb. 19: Im Herbst reifen die Früchte des Granatapfelbaumes. (Foto: H. Steinecke) 


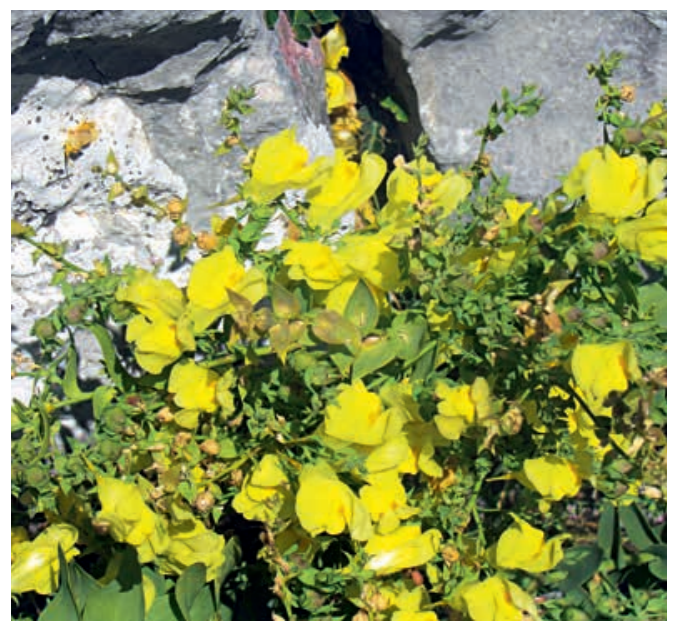

Abb. 20: Balkan-Leinkraut (Linaria dalmatica).

(Foto: H. Steinecke)

sehr trockenen Standorten auf kargem, felsigen Boden ansiedeln.

Hier ist es zu heiß und trocken für größere Gehölze. Man findet an solchen Standorten Rosmarin (Salvia rosmarinus), Salbei (Salvia officinalis) und Lavendel (Lavandula angustifolia). Macchie sowie Felsheiden sind, wenn man sie zur richtigen Zeit aufsucht, äußerst buntblumig, Auffällig sind im Frühsommer Weinraute (Ruta graveolens), verschiedene Thymian- und Gamander-Arten (Thymus spp., Teucrium spp.), Großblütige Prunelle (Prunella grandiflora) und ein Glockenblumengewächs mit ungewöhnlichen, nicht glockenförmigen Blüten, die Hasenglocke (Asyneuma cf. canescens). Im Herbst muss man nur dem süßen Duft nachgehen, dann findet man an Gebüschrändern oft massenhaft Alpenveilchen (Cyclamen hederifolium).

Bis in den Herbst hinein findet man auf felsigen Standorten, auf Mauern und an Wegrändern die stattliche Pyramiden-Glockenblume (Campanula pyramidalis), die wegen ihrer großen und attraktiven Blüten auch als Zierpflanze in Kultur ist. Noch spät im Jahr blüht auch das Dinarische Leinkraut (Linaria dinarica) mit seinen leuchtend gelb gefärbten, lang gespornten Blüten. An kargen Standorten findet man verschiedene Orchideen-Arten, z. B. die Ziegen-Riemenzunge (Himantoglossum

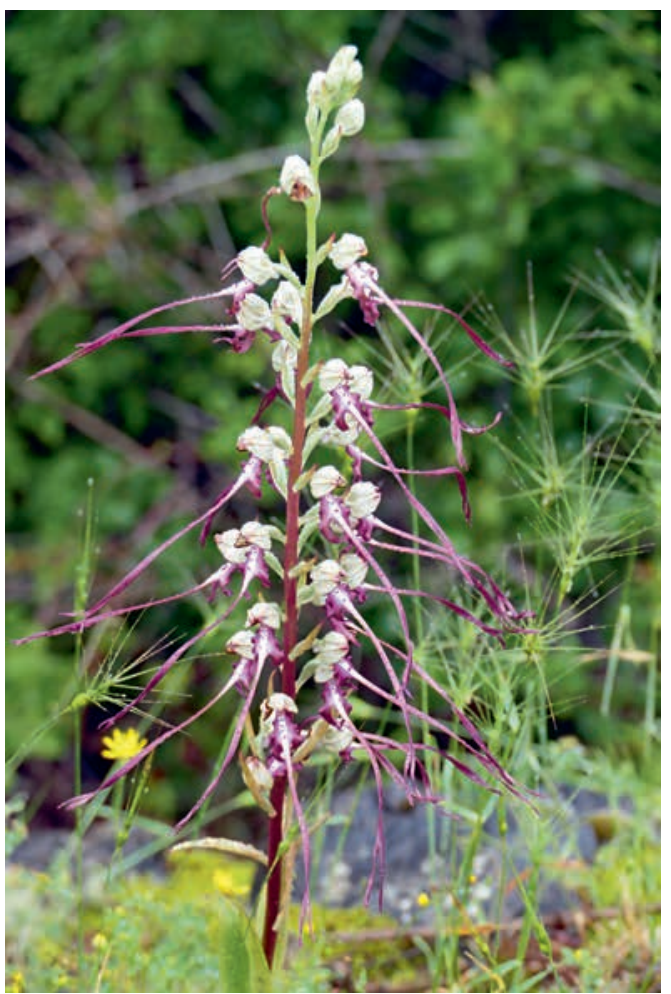

Abb. 21: Ziegen-Riemenzunge (Himantoglossum caprinum). (Foto: H. Steinecke)

caprinum), Hummel-Ragwurz (Ophrys holoserica), Schmetterlings-Knabenkraut (Anacamptis papilionacea), Pyramiden-Knabenkraut (Anacamptis pyramidalis), oder Wanzen-Knabenkraut (Anacamptis, coriophora). Kurzlebige einjährige Arten wie

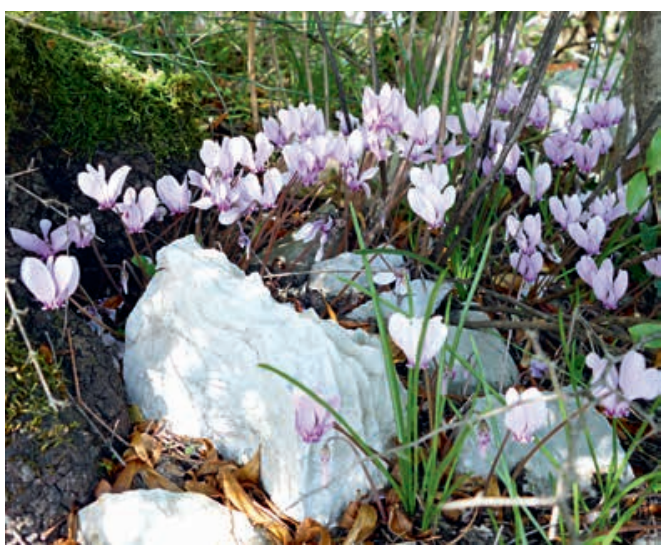

Abb. 22: Ein duftender Herbstblüher ist das Efeublättrige Alpenveilchen (Cyclamen hederifolium). (Foto: H. STEINECKe) 


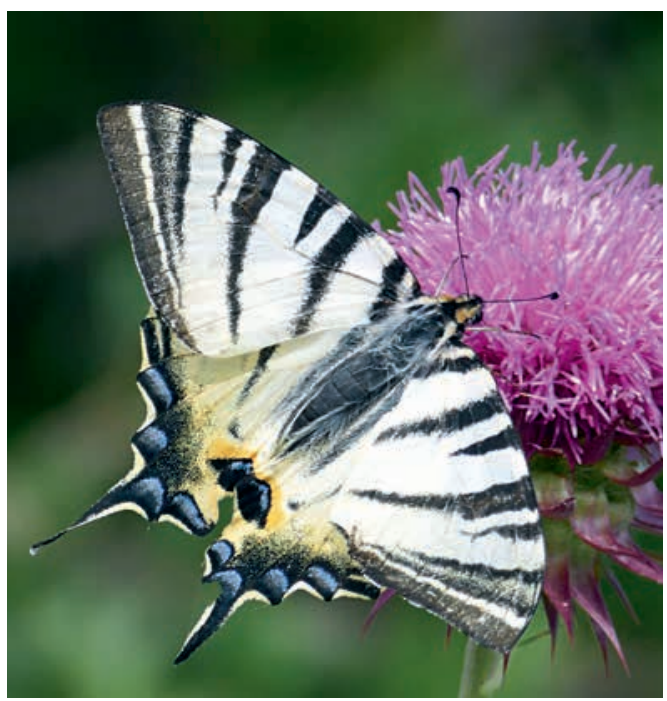

Abb.23:Segelfalter(Iphiclidespodalirius).(Foto:H.STEINECKE)

Jungfer im Grünen (Nigella damascena) oder der Strahlen-Breitsame (Orlaya grandiflora) schmücken Wegränder oft in großen Beständen.

Es wimmelt hier noch von Schmetterlingen, darunter auch die bei uns seltenen Arten Segelfalter (Iphiclides podalirius), Schwalbenschwanz (Papilio machaon) oder Kleiner Schillerfalter (Apatura ilia). Gar nicht mal so selten kommt hier die Gottesanbeterin (Mantis religiosa) vor. An glatten Steinen sind immer wieder die Schaumnester (Ootheken) dieses Insekts zu finden.

Im Bereich von Siedlungen und Höfen gibt es noch traditionelle Trockenmauern. Mit ihnen wurde Weideland für Schafe eingefasst oder generell die Erosion an steilen Hängen eingeschränkt. Im Kulturland wird auch Wein angebaut. Die Qualität des Weines ist sehr gut. Das westliche Hinterland des Sees (Crmnica) ist für den besten Wein in Montenegro bekannt. Die unverputzten Mauern mit ihren vielen Nischen bieten zahlreichen Tieren Schlupfwinkel. Häufig sieht man hier Eidechsen entlanghuschen, mit etwas Glück auch die große, leuchtend grüne Riesen-Smaragdeidechse (Lacerta trilineata). Auch der auf dem Balkan unsere heimische Art ersetzende Weißbrust-Igel (Erinaceus roumanicus) und die Griechische Landschildkröte (Testudo hermanni) finden hier Verstecke.

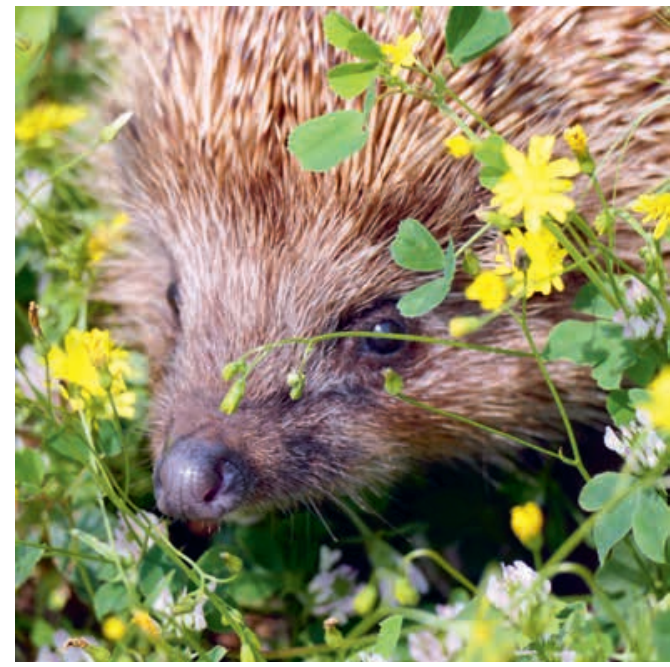

Abb. 24: Ausnahmsweise mal am Tag unterwegs: ein Weißbrust-Igel (Erinaceus roumanicus). (Foto: H. Steinecke)

Noch ist der See ein ziemlich intaktes, großartiges Binnengewässer-Ökosystem ohne Massentourismus. Bedrohungen gibt es schon jetzt, denn das Wasser wird durch angrenzende Landwirtschaft und damit verbundene Düngung stellenweise verschmutzt. Trotzt eines Verbotes wird mit Harpunen oder Strom gefischt. Und leider ist es überall in Montenegro noch üblich, Müll einfach in die Landschaft zu kippen. Es bleibt zu hoffen, dass der Schutzstatus noch besser greift und dieses artenreiche, einzigartige Naturparadies auch für die Zukunft erhalten bleibt.

\section{Literatur und Internetseiten}

Karte Skutari-See 1.55000. Mit Detaillierten Informationen über Flora, Fauna und Geologie. Euronatur. - München.

https://www.montenegro-adria.de/skadarsee-skutarisee/ https://www.euronatur.org/unsere-themen/projektgebiete/projektgebiete-a-z/bojana-buna-delta-und-skutari-see/projekte/ skutari-see/

\section{Anschrift der Autorin und des Autors}

Dr. Hilke Steinecke, Palmengarten Frankfurt,

Siesmayerstr. 61, 60323 Frankfurt.

E-Mail: hilke.steinecke@stadt-frankfurt.de

Dr. Peter Schubert, Universität Mainz, FB Biologie,

Gresemundweg 2, 55128 Mainz,

E-Mail: schubepe@uni-mainz.de 